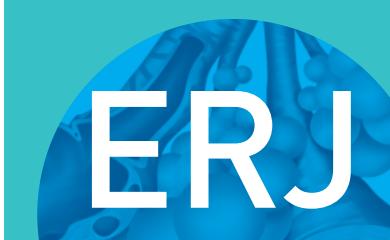

open research

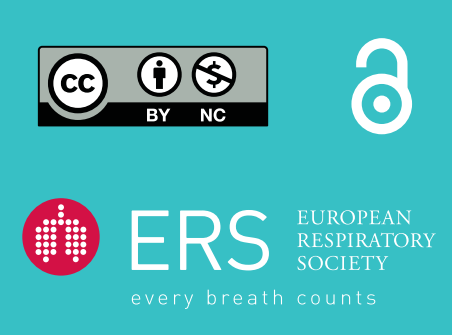

\section{Reflux in idiopathic pulmonary fibrosis: treatment informed by an integrated approach}

\section{To the Editor:}

Despite recent therapeutic advances, the prognosis for patients with idiopathic pulmonary fibrosis (IPF) remains poor. The link with gastro-oesophageal reflux disease (GORD) has been identified as a research priority, as GORD appears to be common in IPF and may be associated with adverse outcomes [1]. GORD is often clinically silent in IPF, so detection is challenging [2].

Traditionally, measurement of reflux has relied on oesophageal $\mathrm{pH}$ probes. Newer probes can demonstrate weakly acid and non-acid reflux through changes in electrical conductivity. Bronchoalveolar lavage fluid (BALF) has been used to provide evidence of aspiration through the detection of gastrointestinal markers [3].

We undertook a pilot study to investigate whether $\mathrm{pH}$-impedance and BALF studies could be combined to assess reflux and aspiration in IPF. We developed a novel "aerodigestive" multidisciplinary team (MDT) approach to support personalised treatment in clinical practice.

Ethical approval was obtained for a prospective observational study (10/H0908/8). Patients diagnosed with IPF according to European Respiratory Society/American Thoracic Society [4] criteria were recruited from the regional interstitial lung disease clinic.

Participants completed the reflux symptom index (RSI), a validated nine-item questionnaire [5]. RSI scores $>13$ are abnormal. For patients taking proton pump inhibitors (PPI), questionnaires were repeated after 2 weeks off therapy, prior to oesophageal physiology and bronchoscopy.

Oesophageal manometry was performed using a water-perfused catheter (MMS, Enschede, the Netherlands). During the study, eight-channel equipment was upgraded to 20-channel high-resolution manometry (HRM). Oesophageal peristalsis and lower oesophageal sphincter function were assessed using established protocols $[6,7]$. The location of the lower oesophageal sphincter was manometrically defined. 24-h ambulatory $\mathrm{pH}$ impedance monitoring was performed using the Ohmega system (MMS). Data were compared to normal ranges in healthy volunteers [8]. The DeMeester score was used as a composite evaluation of distal $\mathrm{pH}[2]$.

Bronchoscopy and lavage were performed to a standardised protocol by a single clinician (IAF). The bronchoscope was wedged in the right middle lobe and $3 \times 60 \mathrm{~mL}$ normal saline instilled. Cell-free BALF supernatants were prepared by centrifugation at $183 \times \mathrm{g}$ for $6 \mathrm{~min}$ at $4^{\circ} \mathrm{C}$ and stored at $-80^{\circ} \mathrm{C}$ for later analysis.

We used a validated, in-house, indirect pepsin ELISA. A monospecific antibody to porcine pepsin was used to measure pepsin and total pepsinogens [9]. Four healthy volunteers recruited from university staff provided BALF samples as controls. We used a spectrophotometric assay to measure total bile acid concentrations (TBA kit; Alere, Stockport, UK).

After spirometry and high-resolution thoracic computed tomography, patients were reviewed in a formal aerodigestive MDT comprising oesophagogastric surgeons, respiratory physicians and anaesthetists.

$@$ ERSpublications

After MDT work-up and review, gastro-oesophageal reflux and pulmonary aspiration were found to be common in IPF patients; surgery was recommended in only $10 \% \mathrm{http}: / /$ ow.ly/ rO3T30lU17o

Cite this article as: Jones R, Krishnan A, Zeybel GL, et al. Reflux in idiopathic pulmonary fibrosis: treatment informed by an integrated approach. ERJ Open Res 2018; 4: 00051-2018 [https://doi.org/ 10.1183/23120541.00051-2018].

Copyright $\odot$ ERS 2018. This article is open access and distributed under the terms of the Creative Commons Attribution NonCommercial Licence 4.0. 
Statistical analysis was performed using Minitab 16 (DataONE, Albuquerque, NM, USA).

79 patients were screened as eligible and invited to participate. 36 patients (27 male) completed all study investigations (October 2010 to June 2014) (figure 1a). Median age was 73 years. 11 patients had previous evidence of GORD, either documented in clinical letters or visualised at endoscopy. Regular medications included PPI $(n=25)$, prednisolone $(n=10 ; 2.5-10 \mathrm{mg}$ dose range) and $\mathrm{N}$-acetylcysteine $(\mathrm{n}=8)$. Subgroup analysis by medication revealed no effect on reflux or aspiration.

Median (range) forced expiratory volume in $1 \mathrm{~s} \mathrm{FEV1}$ was 2.2 (1.4-3.6) L. Median FEV1 was 83.5 (46.8-163.6)\% predicted. Median vital capacity was 2.6 (range 1.5-4.7) L. Median vital capacity was 77.6 (47.9-146.4)\% pred.

The median RSI score for the patients taking PPI was 11 (0-32). The median score off-PPI was 10 (0-39). RSI scores did not correlate with levels of proximal reflux and the effect of PPI withdrawal was inconsistent.

11 patients underwent eight-channel manometry and the remainder underwent HRM. Within the two groups, abnormal physiology was reported in five (45\%) and $15(60 \%)$ patients, respectively. The majority of abnormal tests were consistent with ineffective oesophageal motility (eight-channel) or weak peristalsis (HRM).

All but one subject discontinued PPI therapy. 20 (56\%) patients had a high DeMeester score or prolonged oesophageal acid exposure. In nine patients, impedance analysis revealed high levels of reflux (total, acid or weakly acid). Five of these nine patients had normal $\mathrm{pH}$ traces. Three $(20 \%)$ patients had abnormal proximal oesophageal reflux. Overall, 22 (61\%) patients had a high DeMeester score, a high total number of impedance reflux events or both.

17 (44\%) of the 36 study subjects had pepsin concentrations above the levels recorded in the control population (control range $0-2.3 \mathrm{ng} \cdot \mathrm{mL}^{-1}$ ) (figure $1 \mathrm{~b}$ ). The values for these positive readings ranged from 7 to $44 \mathrm{ng} \cdot \mathrm{mL}^{-1}$. Using the TBA assay, only four out of 36 subjects had detectable levels of bile acids.

In the majority of patients with pathological reflux on ambulatory monitoring, the MDT recommended medical therapy. Lack of fitness for surgery was the commonest reason to recommend against fundoplication. Evidence of reflux from additional investigations was also considered. Antireflux surgery was recommended for three patients; two underwent surgery and the third declined.

We have demonstrated the safety and feasibility of an integrated assessment of gastro-oesophageal reflux and aspiration in an unselected, real-world sample of IPF patients. The pattern of study recruitment helps to illustrate the burden of these invasive tests.

Approximately half of our patients had abnormal levels of reflux at ambulatory monitoring. Our data contrast with higher rates reported previously by RAGHU et al. [10] and SAVARINO et al. [11] (87\% and 83\%, respectively). As in previous reports, clinically silent reflux was common [2]. Reflux symptoms were easier to detect with the use of a structured questionnaire.

The study by SAVArINo et al. [11] is the first to evaluate BALF and saliva/sputum samples from IPF patients for total bile acids. The commercial assay used (Bio-Quant, San Diego, CA, USA) assumes an accurate detection of levels $<1 \mathrm{umol} \cdot \mathrm{L}^{-1}$. The authors concluded that patients with IPF had more bile acids in BALF and saliva than non-IPF patients and controls.

In the current study we used an alternative colorimetric assay, for which we have previously reported superior sensitivity [12]. Only three patients had measurable levels of bile salts and only one of these had readings $>5 \mu \mathrm{mol} \cdot \mathrm{L}^{-1}$. Results from our group and others indicate that colorimetric detection of levels $<5 \mu \mathrm{mol} \cdot \mathrm{L}^{-1}$ may be unreliable $[12,13]$. Overall, we conclude that levels of bile acids were not raised in our patients.

We found elevated levels of pepsin in BALF, indicating that gastric aspiration may occur. This is concordant with the findings of SAVARINO et al. [11] and LEE et al. [14]. Further studies regarding markers of aspiration including pepsin are indicated.

Our study participants had high levels of oesophageal dysfunction at manometry. This may relate to their age, as reference ranges were derived from younger subjects and peristalsis weakens with age [8]. Alternatively, a link between dysmotility and IPF has been suggested [2]. Significant oesophageal hypomotility can compromise swallowing after fundoplication. Detection is mandatory for operative planning and patient consent.

At present, the IPF literature suggests high levels of reflux disease and supports a benefit of antireflux therapy, including fundoplication, for lung health $[10,11,14,15]$. Randomised data are awaited. As we 

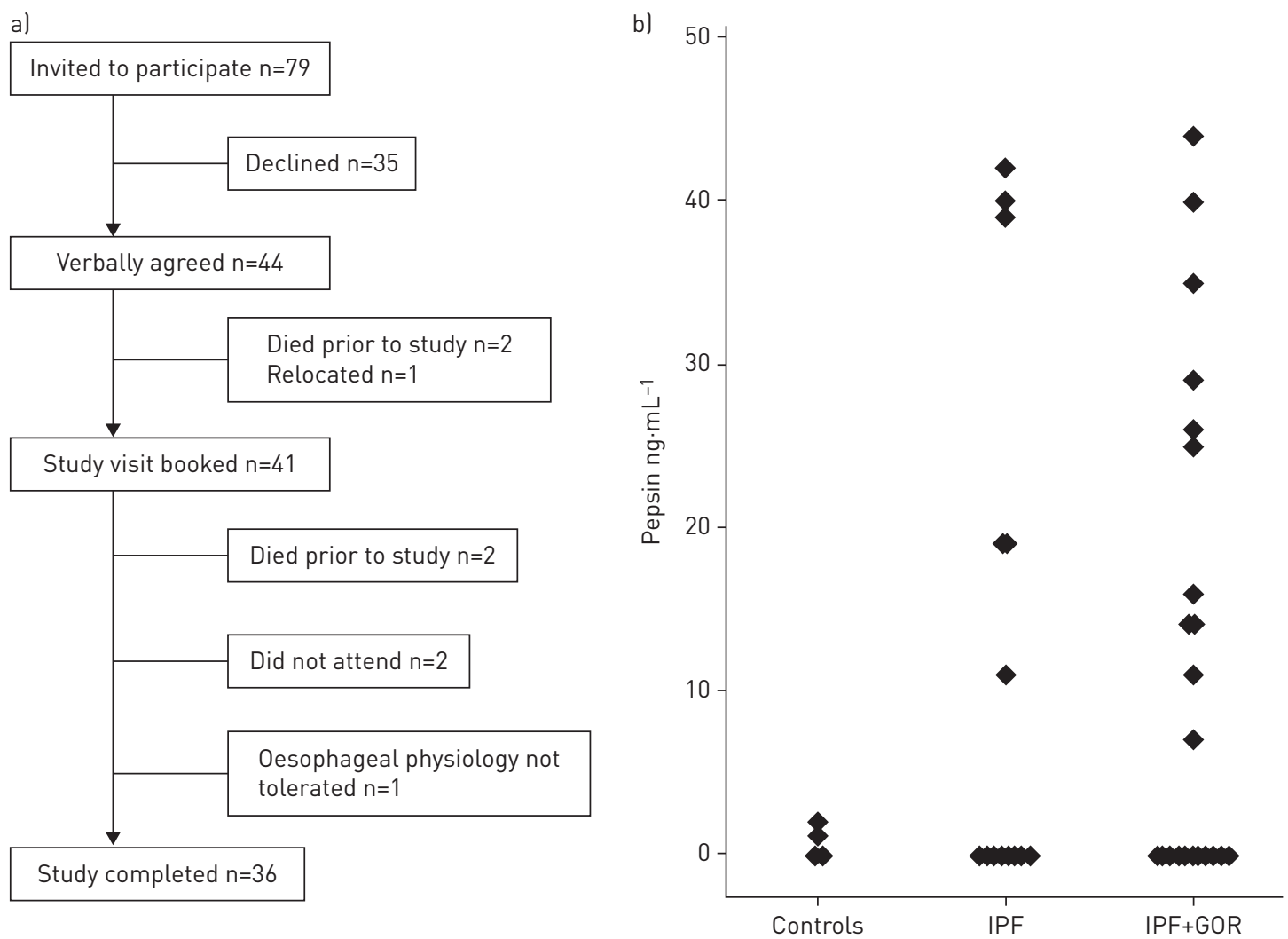

FIGURE 1 a) Flowchart of study recruitment and participation; b) concentrations of pepsin measured in bronchoalveolar lavage fluid from healthy volunteer controls and idiopathic pulmonary fibrosis (IPF) study patients. Study patients with high DeMeester scores (pH assessment) or supranormal total reflux events (impedance assessment) are indicated as IPF+GOR (gastro-oesophageal reflux disease).

learn how best to measure reflux and aspiration in IPF, it is crucial that the risks of surgery in the IPF population are accurately represented. In our institution, after formal MDT review, fundoplication was only recommended for a minority of IPF patients. Antireflux surgery may be appropriate in selected cases, but careful consideration is required in a group of patients who are often elderly with multiple comorbidities.

Rhys Jones ${ }^{1,5}$, Amaran Krishnan ${ }^{1,5}$, Gemma L. Zeybel ${ }^{2}$, Emily Dookun ${ }^{2}$, Jeffrey P. Pearson ${ }^{2}$, A. John Simpson ${ }^{3}$, S. Michael Griffin ${ }^{1}$, Chris Ward $\circledast^{2,3,6}$ and Ian A. Forrest ${ }^{4,6}$

${ }^{1}$ Northern Oesophago-Gastric Unit, Royal Victoria Infirmary, Newcastle-upon-Tyne, UK. ${ }^{2}$ Institute of Cell and Molecular Biosciences, The Medical School, Newcastle University, Newcastle-upon-Tyne, UK. ${ }^{3}$ Institute of Cellular Medicine, The Medical School, Newcastle University, Newcastle-upon-Tyne, UK. ${ }^{4}$ Dept of Respiratory Medicine, Royal Victoria Infirmary, Newcastle-upon-Tyne, UK. ${ }^{5}$ Joint first authors. ${ }^{6}$ Joint senior authors.

Correspondence: Ian A. Forrest, Dept of Respiratory Medicine, Royal Victoria Infirmary, Queen Victoria Road, Newcastle-upon-Tyne, NE1 4LP, UK. E-mail: ian.forrest@nuth.nhs.uk

Received: April 042018 | Accepted after revision: Sept 112018

Conflict of interest: None declared.

Support statement: This work was jointly funded by Innovate UK and the Northern Oesophago-Gastric Fund. Funding information for this article has been deposited with the Crossref Funder Registry.

\section{References}

1 NICE. CG163 Idiopathic Pulmonary Fibrosis: NICE Guidance. National Institute of Clinical Excellence, 2013.

2 D'Ovidio F, Singer LG, Hadjiliadis D, et al. Prevalence of gastroesophageal reflux in end-stage lung disease candidates for lung transplant. Ann Thorac Surg 2005; 80: 1254-1260. 
3 Meyer KC, Raghu G, Baughman RP, et al. An official American Thoracic Society clinical practice guideline: the clinical utility of bronchoalveolar lavage cellular analysis in interstitial lung disease. Am J Respir Crit Care Med 2012; 185: 1004-1014.

4 Joint ATS/ERS Working Group. American Thoracic Society. Idiopathic pulmonary fibrosis: diagnosis and treatment. International consensus statement. American Thoracic Society (ATS), and the European Respiratory Society (ERS). Am J Respir Crit Care Med 2000; 161: 646-664.

5 Belafsky PC, Postma GN, Koufman JA. Validity and reliability of the reflux symptom index (RSI). J Voice 2002; 16: 274-277.

6 Bredenoord AJ, Fox M, Kahrilas PJ, et al. Chicago classification criteria of esophageal motility disorders defined in high resolution esophageal pressure topography. Neurogastroenterol Motil 2012; 24: Suppl. 1, 57-65.

$7 \quad$ Spechler SJ, Castell DO. Classification of oesophageal motility abnormalities. Gut 2001; 49: 145-151.

8 Zerbib F, des Varannes SB, Roman S, et al. Normal values and day-to-day variability of 24-h ambulatory oesophageal impedance-pH monitoring in a Belgian-French cohort of healthy subjects. Aliment Pharmacol Ther 2005; 22: 1011-1021.

9 Stovold R, Forrest IA, Corris PA, et al. Pepsin, a biomarker of gastric aspiration in lung allografts: a putative association with rejection. Am J Respir Crit Care Med 2007; 175: 1298-1303.

10 Raghu G, Freudenberger TD, Yang S, et al. High prevalence of abnormal acid gastro-oesophageal reflux in idiopathic pulmonary fibrosis. Eur Respir J 2006; 27: 136-142.

11 Savarino E, Carbone R, Marabotto E, et al. Gastro-oesophageal reflux and gastric aspiration in idiopathic pulmonary fibrosis patients. Eur Respir J 2013; 42: 1322-1331.

12 Parikh S, Brownlee IA, Robertson AG, et al. Are the enzymatic methods currently being used to measure bronchoalveolar lavage bile salt levels fit for purpose? J Heart Lung Transplant 2013; 32: 418-423.

13 Klokkenburg JJ, Hoeve HL, Francke J, et al. Bile acids identified in middle ear effusions of children with otitis media with effusion. Laryngoscope 2009; 119: 396-400.

14 Lee JS, Song JW, Wolters PJ, et al. Bronchoalveolar lavage pepsin in acute exacerbation of idiopathic pulmonary fibrosis. Eur Respir J 2012; 39: 352-358.

15 Allaix ME, Fisichella PM, Noth I, et al. Idiopathic pulmonary fibrosis and gastroesophageal reflux. Implications for treatment. J Gastrointest Surg 2014; 18: 100-104. 